\title{
Creative Class, Creative Industries, Creative CITY. EIN MUSIKPOLITISCHES PARAdIGMA
}

\author{
Alenka Barber-Kersovan
}

\section{Was ist Creativity?}

Der Begriff »Kreativität« hat - insbesondere in seiner englischen Variante »Creativity« - wieder Konjunktur: als ein Modebegriff, als die Bezeichnung für alles, was in gewisser Hinsicht als neu betrachtet werden kann und vor allem als ein politisches Zauberwort, mit dessen Hilfe man der dramatischen Veränderungen in allen Lebensbereichen Herr zu werden hofft. Die Rhetorik der zahlreichen Schriften zu diesem Phänomen zeichnet sich durch die Ästhetisierung des Diskurses, Optimismus und Zukunftsgläubigkeit aus. Hinter der verführerisch klingenden Beredsamkeit verbirgt sich allerdings die harte Realität des neo-liberalen Post-Fordismus und entlarvt Creativity als einen ökonomischen Faktor mit vermeintlich unbegrenztem Wertschöpfungspotential.

Auch in Bezug auf den Sound und die City kreist der Diskurs um den Begriff Creativity vorwiegend um Sachverhalte wie Wettbewerbsfähigkeit und Innovationskraft von Städten unter dem Aspekt ihrer Wirtschaftlichkeit. Dabei wird eine Argumentationskette verfolgt, die sich aus den Schlüsselbegriffen Creative Class, Creative Industries und Creative City ergibt und die ins Zentrum ihrer Überlegungen die Rolle der populären Musik für die urbane Regeneration de-industrialisierter Stadtteile und das Image-Design städtischer Konglomerate stellt. Diese Argumentationskette erfreut sich zurzeit einer lebhaften Umsetzung theoretischer Axiome in politische Entscheidungen und in konkrete Projekte.

Die Bezeichnung Creative Class wurde durch die Abhandlung The Rise of the Creative Class and how it is Transforming Work, Leisure, Community and Everyday Life des amerikanischen Wirtschaftswissenschaftlers Richard Florida (2004b) im Umlauf gebracht. Das äußerst populäre Buch basiert auf der weitläufigen Annahme, dass wir uns zurzeit an einem historischen Wen- 
depunkt befinden: Das Zeitalter der Industrialisierung weiche einer Ära der Kreativität. Die wichtigsten Ressourcen dieser neuen Epoche bildeten nicht mehr Rohstoffe, sondern Informationen, die wichtigsten Produkte nicht mehr die industrielle Massenware, sondern Ideen und Bedeutungen, und die wichtigsten Produktionsmittel nicht mehr die Maschinen, sondern die Vorstellungskraft der Creative Class.

Creativity wird von Florida als »the ability to create meaningful new forms« (ebd.: 5) definiert. In diesem Punkt deckt sich sein Verständnis weitestgehend mit den älteren definitorischen Ansätzen, die Kreativität als einen intrapsychischen Vorgang auslegten, verbunden mit Sachverhalten wie divergentes, produktives oder laterales Denken. Auch vertritt Florida die nun bereits allgemein akzeptierte These, zwischen den unterschiedlichen Ausprägungsformen des kreativen Verhaltens gebe es keine qualitativen, sondern lediglich quantitative Unterschiede. Sein Konzept schließt deshalb so genannte Genies wie Mozart und Beethoven zwar nicht aus, doch bilden den Schwerpunkt schöpferischer Prozesse im Sinne von Florida eher kleinere Umformungen des Bekannten, die sich vorwiegend in Objekten des täglichen Gebrauchs materialisieren.

\section{Creative Class}

Mit der Annahme, der Träger der Kreativität sei nicht ein isoliertes Individuum, sondern eine bestimmte soziale Gruppe mit spezifischen Eigenschaften, die so genannte Creative Class, weicht Florida von den gängigen theoretischen Ansätzen ab. Diese Klasse setze sich aus hoch qualifizierten Personen aus unterschiedlichsten Berufen zusammen: Musiker, Künstler und Wissenschaftler gehörten ebenso dazu wie Lehrer und andere "professionals«, deren Arbeit originelle Lösungen anstehender Probleme voraussetzt.

Der Arbeitsinhalt der Creative Class ist deshalb die Content Production, d.h. die Produktion von künstlerischen, wissenschaftlichen und sonstigen Inhalten mit innovativem Charakter - auch dieser Aspekt wurde bereits von der bisherigen Kreativitätsforschung betont. Ein Unterschied zu den Kreativen der Vergangenheit ergebe sich allerdings daraus, so Florida (ebd.: 40), dass sich zwar auch die Vertreter der Creative Class durch Individualismus und ein gewisses Maß an Nonkonformität auszeichnen. Sie wiesen aber zudem ein starkes Bedürfnis nach Organisation und einer spezifisch strukturierten Umgebung auf, die es ihnen erlaubt, kreativ zu sein, und die ihre Leistungen - kleine Veränderungen ebenso wie eine gelegentliche sgroße Idee $<$ entsprechend würdigt. 
Ein weiteres Wesensmerkmal liege darin, dass zwar auch die Creative Class intrinsisch motiviert ist und gerne das tut, worin sie gut ist und wofür sie die entsprechenden sozialen Gratifikationen erhält. Als ein Kind der Creative Economy (vgl. dazu Howkins 2002) schöpfe sie, laut Florida (2004b: XIII), aber nicht primär zum Selbstzweck, sondern setze die kreative Arbeit gezielt ein, um mit ihr den Lebensunterhalt zu verdienen. Die kreative Leistung sei deshalb eine ökonomische Ressource mit einem expliziten Wettbewerbscharakter und bilde häufig auch das einzige Kapital, das die Beteiligten besitzen und unter bestimmten Bedingungen dem Arbeitsmarkt anzubieten haben. Aus diesem Grund gestalten sie vorwiegend marktfähige Erzeugnisse, deren Erlös ihnen neben der Deckung von Grundbedürfnissen auch das Führen eines abwechslungsvollen Lifestyles erlaubt (vgl. ebd.: 13).

\section{Creative City}

Um sich entsprechend zu entfalten, braucht die Creative Class nach Florida (ebd.: 7) abwechslungsreiche und animierende Rahmenbedingungen, wie diese in einem pulsierenden urbanen Milieu zu finden sind. Das Verhältnis zwischen der Creative Class und der City ist deshalb symbiotisch. Die Wissensgesellschaft der Creative Era ist eine urbane Gesellschaft (vgl. Landry 2000: XIII), und die Creative Class ist eine durch und durch urbane Spezies, die mit ihrem unkonventionellen, sich durch Toleranz, Offenheit und Kommunikationsfreudigkeit auszeichnenden Lebensstil (vgl. Florida 2002b: 9) Wesentliches zum urbanen Flair beiträgt.

Doch dies ist nur eine Seite der Medaille, denn falls man mit John Howkins davon ausgeht, dass in der Creative Era Kreativität und Innovation die treibenden gesellschaftlichen und ökonomischen Kräfte darstellen, ist für eine Stadt die Anwesenheit der Creative Class auch von enormer wirtschaftlicher Bedeutung. Falls die Zukunft der Wissens- und Informationsgesellschaft in ihrer innovativen Kraft liegt (vgl. Howkins 2002: 23), ist es sowohl im Interesse einer Gesellschaft als auch einer Metropole, in das kreative Kapital zu investieren und die Creative Class an sich zu binden. In Bezug auf die Städteplanung solle dies durch die Gestaltung von entsprechenden kreativitätsfördernden Milieus geschehen, worunter unter anderem eine lebendige Musik- und Kunstszene verstanden wird (vgl. Florida 2004a: 84 und 2004b: 55).

Die Creative Class hat - folgt man Florida - für eine Stadt noch weitere Vorteile. Die Kreativitätsklammer funktioniere identitätsstiftend und stärke nicht nur die soziale Kohärenz der Stadtbewohner, sondern trage auch 
Wesentliches zum Image-Design einer Stadt bei. Zudem sichere eine lokal gefärbte Content Production entsprechende Marktanteile auf dem globalen Umschlagsplatz und erhöhe die ökonomischen Chancen einer Stadt. Im Rahmen einer postindustriellen Gesellschaft sei deshalb eine Creative City zugleich auch eine Competitive City, die im Wettbewerb mit anderen urbanen Konglomeraten ihr symbolisches Kapital im Kampf um die ökonomische Vormacht einsetzt.

\section{Die Rolle der Pariser Bohème für die kulturelle Konstruktion der französischen Metropole}

So originell, wie es die in zahlreichen Variationen um den Begriff Creativity oszillierende Terminologie suggeriert, sind die Zusammenhänge zwischen der kreativen Klasse und der Stadt allerdings nicht. Ähnliches ist auch in der Abhandlung Paris. Die kulturelle Konstruktion der französischen Metropole von Klaus Schüle (2003) nachzulesen. Schüle geht in seinem kulturwissenschaftlichen Beitrag zur Urbanisierungsgeschichte davon aus, dass Kultur einen wesentlichen Faktor dessen darstellt, was die Eigenart von Paris ausmacht. Eine wichtige Rolle spielen dabei die Quartiere Montmartre, Quartier Latin und Saint Germain, die Schüle als spezifische, durch Kultur geprägte Lebensräume vorstellt. Seine These belegt er mit der Darstellung der Lebensweise zweier kreativer Gruppierungen, der Avantgarde und der Bohème.

Schüles Ausführungen sind vorwiegend historisch. Gedanklich knüpfen sie an Karl Marx und an Walter Benjamins Abhandlung Das Paris des Second Empire bei Baudelaire an, deren erstes Kapitel die Überschrift »Die Bohème « trägt. Demnach bilden die erwähnten Künstlerviertel den Lebensraum einer »Klasse «, im »Einzelnen mehr vom Zufall als von ihrer Thätigkeit abhängige[n] Existenz« lebend, deren »einzige fixe Stationen die Kneipen der Weinhändler sind« (zit. n. Schüle 2003: 20). Dieser intellektuell privilegierten und zugleich sozial benachteiligten Klasse gehörten neben den Künstlern aller Kunstrichtungen auch viele Musiker an. Wie aus der folgenden Skizze aus dem Leben der Bohème nach dem Zweiten Weltkrieg hervorgeht, wurde deshalb in den Szenenkneipen stets auch sehr viel Musik gemacht und gehört. Boris Vian schrieb:

»1925 trafen sich die Maler in weitläufigen Ateliers. 1945 wohnten die jungen Leute in Mansarden, die drei Quadratmeter maßen. Sie suchten nach Orten, wo sie sich treffen konnten. Zuerst war es die >Bar Vert<, wo Bernard Lucas, ein Musikkritiker[,] Platten mit klassischer Musik vorstellte. Man muß wirk- 
lich festhalten, daß die Jahre 1945-1950 eine erstaunliche Revolution hervorbrachten: die der Platten und Langspielplatten. Man konnte doch nicht immerzu bei Beethoven bleiben, der Jazz rief uns. Aus diesem Grunde eröffnete Guionett das >Tabou<. Dort sah man Juliette Gréco, die damals eine junge Schauspielerin war, und die auch eine Rolle gespielt hatte und zwar eine richtige, in der Résistance. Sie trug Gedichte vor, zusammen mit Anne-Marie Cazalis, die den Paul-Valéry-Lyrik-Preis gewonnen hatte. Jeder konnte auf der Trompete improvisieren, tanzen ohne zu bezahlen, vortragen, was ihm so durch den Kopf ging «(Boris Vian; zit. n. Schüle 2003: 52f.).

An einer anderen Stelle ist bei Schüle Folgendes nachzulesen:

»Die Musik also ist neben den eingeschränkten Wohn- und Lebensbedingungen der zweite große Unifikator der sehr diffusen intellektuellen Szene gewesen. Natürlich spielte die Musik nicht nur im >quartier latin großen des französischen und internationalen Jazz wie Django Reinhardt, Stéfane Grapelli, Sidny [sic] Bechet usw. spielten durchaus auch an anderen Orten). Wie im Deutschland der Nachkriegszeit hat aber der Jazz in Frankreich eine ganze Generation geformt, ganze Stadtviertel geprägt [...]. Es war aber im Unterschied zu Deutschland - keineswegs die Jazz-Szene allein, die in den Cafés, Kellern und Kneipen den Ton angab. Es war die sganze organisierte Verrücktheit<, die an diesen Orten aufeinander traf. Hier wurden Verse von Prévert vorgetragen [...], hier sang die Gréco, dort spielte eine ausländische Musikgruppe auf, hier traten >les Frères Jaques< auf, dort sang Jacques Douai >Les feuilles mortesく. Der Jazz aber dominierte die Szene« (Schüle 2003: 56).

Der Lifestyle der Bohème war bunt und laut, und er wurde bereits zu damaliger Zeit medial ausgeschlachtet. Sensationslüsterne Reporter hatten ein großes Interesse an den künstlerischen Nonkonformisten, und auch die Bohème selbst sorgte für Aufsehen, indem sie sich entsprechend schrill in Szene setzte (vgl. ebd.: 32). Ihre organisierte Verrücktheit konnte sie zwar nicht in bare Münze umsetzen. Sie prägte aber bestimmte Vorstellungen über ihr extravagantes Leben, die wiederum auf jene Stadtteile abfärbten, die von ihr in Besitz genommen wurden. Die meisten der einstigen Protagonisten sind längst tot. Was allerdings noch weiterhin lebt, ist der Mythos von Paris, der sich tausendfach in der bildenden Kunst, im Film, in der Literatur, aber auch in den zahlreichen Songs und Chansons reproduziert - und vor allem noch Generationen später die Kassen jener füllt, die am ImageDesign der einstigen Künstlerviertel nicht beteiligt waren und die nun die einstige Magie dieser Orte als billige Souvenirs an Schaulustige aus aller Welt verscherbeln. »Kultur hat Konjunktur. Schon seit langem«, schlussfolgert Klaus Schüle. »Kultur rechnet sich. Schon seit langem. Kultur zieht den Tourismus an, ist ein Standortfaktor $\ll$ (ebd.: 12). 


\section{Creative Class als Neo-Bohème}

In Bezug auf das behandelte Thema muss hervorgehoben werden, dass auch Schüle die Bohèmiens nicht primär als Individuen, sondern als eine Klasse behandelt. Folgerichtig reduziert er ihre schöpferische Arbeit auch nicht auf ein einfaches Verhältnis zwischen dem Künstler und seinem Werk. Dass es ein derartiges Verhältnis gibt und dass dieses für das Zustandekommen eines Buches, eines Bildes oder eines Musikstückes von enormer Wichtigkeit ist, lässt sich natürlich nicht verneinen. Aber dies ist nicht das einzige Verhältnis, in dem sich kreative Individuen befinden. Sie sind auch in diverse Kommunikationsnetzwerke eingebunden und leben in spezifischen kunst- bzw. musikzentrierten sozialen Milieus (vgl. Landry, 2000: 133-135). Und diese Milieus wiederum bilden nicht nur eine wichtige Inspirationsquelle, sondern sie stellen auch eine unabdingbare Voraussetzung für die Produktion und Distribution (und damit für die Vermarktung) ästhetischer Artefakte dar.

Die Creative Class im Sinne von Florida trägt somit viele Gesichtszüge des einstigen Künstlerlebens, und sie wird auch oft als Neo-Bohème (vgl. dazu Lloyd 2006) bezeichnet. Um diesen Sachverhalt zu betonen, entwickelte Florida (2004b: 260) einen »Bohemian Index«, der die Anzahl der Künstler in einer Stadt wiedergibt und anhand dessen er ein Ranking US-amerikanischer Städte als Creative Cities unternahm.

Ein Unterschied zwischen der Bohème und der Neo-Bohème liegt allerdings darin, dass der künstlerische Lifestyle, früher einer überschaubaren Anzahl Kreativer vorbehalten, nun bereits von Millionen (vorwiegend) junger Menschen gelebt wird. Ferner charakterisiert die Creative Class die Verbindung zweier zuvor als unvereinbar betrachteten Eigenschaften: die bürgerliche, neo-liberale Arbeitsethik mit dem ungezwungenen Habitus der Bohème. Die Creative Class mag sich deshalb nonchalant geben und sie mag ihre Arbeit durchaus als persönliche Erfüllung, Selbstverwirklichung oder identitätstiftende Betätigung empfinden, aber im Gegensatz zu der historischen Bohème, die die Kunst der Muse zelebrierte, ist die Neo-Bohème nicht idealistisch, sondern realistisch orientiert und stets bereit, hart zu arbeiten, um auch die entsprechenden finanziellen Zielsetzungen zu erreichen (vgl. ebd.: 192ff.). 


\section{Creative Industries}

Vertreter der Creative Class decken ein breites Spektrum beruflicher Qualifikationen $a b$ und bringen unterschiedliche künstlerische und intellektuelle Inhalte hervor. In Bezug auf die ästhetische Produktion dürfen der Verbindung zwischen dem künstlerischen Streben und der kommerziellen Tüchtigkeit am ehesten jene Inhalte entsprechen, die unter dem Begriff »Creative Industries« (vgl. dazu Hartley 2005) zusammengefasst werden. Laut dem von der britischen Regierung bereits in 1998 ausgearbeiteten "Creative Industries Mapping Document « gehören dazu alle Tätigkeiten, die ihren Ursprung in der individuellen Kreativität haben und die das Potential besitzen, durch die Anwendung intellektueller Fähigkeiten und spezifischer Fertigkeiten Wohlstand und Beschäftigung zu schaffen. ${ }^{1}$

Im Einzelnen werden zu den Creative Industries die folgenden ästhetischen Sparten gezählt: Architektur, Bildende Kunst, Darstellende Kunst, Unterhaltung, Fernsehen, Film, Video und andere audiovisuelle Medien, Graphik und Design, Live Musik und Tonträgerproduktion, Mode, Rundfunk und Internetübertragungen, Schreiben und Publizieren sowie Werbung. ${ }^{2}$ Die meisten der erwähnten Bereiche haben auf irgendeine Art und Weise mit Musik zu tun. Ein wichtiges Merkmal bilden ferner Überschneidungen mit dem Telekommunikationssektor sowie neuartige Produktions- und Distributionsformen in Folge der digitalen Revolution (Internetwirtschaft).

Die erwähnten ästhetischen Bereiche sind natürlich nichts Neues. Neu dürfte aber die Art und Weise sein, in der die Interdependenz zwischen Kunst, Kultur, Wirtschaft und Arbeitsmarkt begründet wird. Diese stellt insofern einen Bruch mit dem herkömmlichen Kulturverständnis dar, indem sie, statt dem »rationalen und funktionalen Wesen der Wirtschaft « das »soziale und emotionale Wesen der Kultur « gegenüber zu stellen, diese bislang vielfach als unvereinbar angesehenen Sachverhalte miteinander verknüpft. Die Spannung zwischen Kunst und Kommerz, wie sie etwa von Horkheimer und Adorno thematisiert wurde (1971: 108ff.), wird dadurch aufgehoben, das Verständnis von Kunst und Kultur um eine ökonomische Variable erweitert. ${ }^{3}$ Folgerichtig ist aus der Perspektive der Creative Industries ein Künstler im Sinne der Creative Class nicht nur ein Erzeuger kulturellen, sondern

1 Vgl. http://www.labforculture.org/en/resources/research_in_focus/no_1_the_ culture_industries_in_europe/the_creative_industries_mapping_document_200 1_1 (Zugriff am 27.5.2007).

2 Vgl. ebd.

3 Eine kritische Evaluation dieses Konzeptes unternahm unter anderen Gerald Raunig (2007). 
zugleich auch ökonomischen Kapitals. Vom Creative Clusters Network, einem internationalen Zusammenschluss kommerziell orientierter Initiativen zur Förderung der Kultur, wurde dieser Sachverhalt folgendermaßen zusammengefasst:

»From the creative industry perspective the artist is a generator of economic value. To many people this is a strange and unwelcome idea. We are used to thinking of economic wealth and power as something totally distinct from cultural wealth and power. «4

Ebenso wie Klaus Schüle in seinen Ausführungen über Paris, betont deshalb auch das Creative Clusters Network, dass sich die Kultur >rechnet<, und begründet seine Schlussfolgerung »Culture is Big Business unter anderem damit, dass zu den erfolgreichsten Unternehmen nicht industrielle Großbetriebe, sondern Medienkonzerne, Verlage, Vertreter der Unterhaltungsindustrie und Designer von Computerspielen zählen. Der erwähnte Arbeitssektor gehöre weltweit zu den Schlüsselbranchen und erfreue sich jährlicher Zuwachsraten zwischen 6 und 20 Prozent. $^{5}$

\section{Ergebnisse empirischer Untersuchungen}

\section{Schwerpunkt Liverpool}

Der Frage, wie sich die erwähnten Zusammenhänge konkret gestalten und welche Rolle sie für die Creative City spielen, wurde in mehreren Forschungsprojekten nachgegangen. Vom Institute of Popular Music an der University of Liverpool wurden zu diesem Thema drei aufeinander folgende Forschungsvorhaben durchgeführt. Das erste Projekt befasste sich mit der Populären Musik des 20. Jahrhunderts in Liverpool. Das zweite trug den Titel Music Policy, the Music Industry and Local Economic Development und umfasste eine vergleichende Analyse musikpolitischer Entwicklungsstrategien in drei englischen Städten. Das Projekt Popular Music, Tourism and Urban Regeneration untersuchte das Verhältnis zwischen der Stadt und der Musik in Liverpool und New Orleans, in zwei als Musikstädte bekannten urbanen Konglomeraten, die den Kultur-Tourismus als einen der wichtigsten Bereiche der urbanen Regeneration betrachten.

\footnotetext{
4 Zit. n. http://www.creativeclusters.com/modules/eventsystem/?fct=eventmen us\&action=displaypage\&id=34 (Zugriff am 20.6.2007).

5 Ebd.
} 
Die wichtigsten Ergebnisse der genannten Studien wurden in der Abhandlung Decline, Renewal and the City in Popular Music Culture: Beyond the Beatles von Sara Cohen (2007) zusammengefasst. Die ethnographischen Detailanalysen, basierend auf ausgewählten Fallbeispielen, verwiesen darauf, dass das Musikleben einer Stadt von einer großen Anzahl musikalischer Akteure (Musiker, Manager, Fans, Veranstalter, Produzenten und Distribuenten von Tonträgern und anderen musikbezogenen Waren, Vertreter der Tourismusbranche und der Kommunalpolitik etc.) getragen wird. Zu den wesentlichsten Charakteristika der Musik in einem urbanen Milieu gehören deshalb die beinahe unüberschaubare Vielfalt musikalischer Handlungsweisen, die Komplexität der Beziehungen zwischen den einzelnen an der Gestaltung des Musiklebens beteiligten Individuen, Gruppen und Institutionen, die - aus unterschiedlichen Motiven heraus agierend - unterschiedliche und teilweise auch entgegengesetzte Zielsetzungen verfolgen, sowie die beschleunigte Dynamik gravierender Veränderungen. Weitere Fragestellungen betrafen das Verhältnis der politischen Entscheidungsträger zu den einzelnen Segmenten der Musikszene, die Art der angewandten Förderstrategien seitens städtischer und staatlicher Instanzen sowie deren Auswirkungen sowohl auf die Beteiligten als auch auf das Image-Design von Städten, insbesondere von Liverpool als international anerkannter Music City. Eindeutige Schlussfolgerungen, die als Richtlinien für die künftige Kulturpolitik dienen könnten, konnten laut Cohen jedoch nicht gezogen werden, da die Einschätzung der Situation von der Position der jeweiligen Akteure im Gesamtsystem des städtischen Musiklebens abhängt und dementsprechend unterschiedlich ausfällt (vgl. Cohen 1991 und 2007).

\section{Schwerpunkt Manchester}

Ein weiteres Forschungsprojekt wurde im Rahmen des British Cities Programme vom Manchester Institute for Popular Culture an der Manchester Metropolitan University durchgeführt und trug den Titel Cultural Industries and the City: Innovation, Creativity and Competitiveness. Die Autoren Justin O'Connor und Mark Banks untersuchten die Stadt als wettbewerbsfähiges, innovatives Milieu und stellten die Frage, inwieweit Cultural Industries von diesem Milieu profitierten und was sie dazu sowohl in materieller als auch in symbolischer Hinsicht selbst beitrügen. Die wichtigsten Ergebnisse lassen sich wie folgt zusammenfassen:

1. In der Manchester City Area sichert der Kultursektor $6 \%$ aller Arbeitsplätze, und die Beschäftigung in diesem Bereich steigt. Auch die Revitalisierung des Stadtzentrums wird weitgehend auf die steigende kulturelle 
Produktion bzw. auf den steigenden Konsum in diesem Bereich zurückgeführt.

2. Auf der sozialen Ebene zeichnet sich der Kulturbereich durch Partnerschaft, Networking, Kreativität und Kooperation aus, was sich positiv auf die Steigerung der Produktivität auswirkt.

3. Globalisierungsprozesse haben auf dieses Milieu einen zwiespältigen Einfluss. Einerseits untergraben sie seine durchaus fragile ökonomische Basis. Andererseits regen sie aber die Produktion von neuen Kulturprodukten an, fördern neue geschäftliche Beziehungen und stimulieren eine risikofreundliche Geschäftsattitüde.

4. Die meisten der untersuchten Unternehmen sind kleine Firmen, die sich einer lokal gefärbten Produktion verschrieben haben. Einige unter ihnen expandieren. Andere wiederum, insbesondere in den Bereichen Musik, Promotion, Neue Medien und Design, bleiben zwar Kleinunternehmer, sie ziehen aber weitere Kleinunternehmen an und tragen deshalb nicht nur zur Verdichtung kultureller Netzwerke, sondern auch zum ökonomischen Wachstum sowie zur kulturellen Diversivität und dementsprechend auch zum urbanen Charakter der Stadt Manchester bei.

5. Die Arbeit in den Kultursegmenten Musik, Mode, Kunst und Design verbindet ökonomische und kulturelle Werte und hilft den Städten, neue Potentiale zu generieren und den strukturellen Veränderungen sowohl auf der lokalen als auch auf der globalen Ebene standzuhalten.

6. Creative Industries spielen eine vitale Rolle bei der Promotion einer Stadt als Competitive City, da sie als ein Indikator für die Dynamik, Flexibilität und Kreativität der Stadt als Ganzes angesehen werden. Aus diesem Grund solle innen eine zentralere Rolle in der Stadt- und Kommunalpolitik eingeräumt werden (vgl. O'Connor/Banks 2000).

\section{Schwerpunkt Wien}

Bei der Studie Untersuchung des ökonomischen Potentials der Creative Industries in Wien handelt es sich um den Endbericht der Österreichischen Kulturdokumentation in Zusammenarbeit mit dem Internationalen Archiv für Kulturanalysen, dem Ministerium für Wirtschaft und Forschung und Mediacult, dem Forschungsinstitut für Medien und Kultur unter der Schirmherrschaft der UNESCO. Sie wurde von der Stadt Wien, der Wirtschaftskammer Wien und den Filmfonds Wien in Auftrag gegeben und aus den Mitteln des Europäischen Fonds für Regionale Entwicklung finanziert. 
Auch diese Studie fasst unter dem Oberbegriff Creative Industries eine Vielfalt von heterogenen Wirtschaftszweigen zusammen. Die Zahl von Unternehmen, in deren Zentrum kreatives Gestalten steht, ist beachtlich. Es wurden insgesamt 17.948 kreative Betriebe mit ca. 120.000 Arbeitnehmern ermittelt: Das sind rund $14 \%$ aller in Wien beschäftigten Personen. Mit einem durchschnittlich 6\%-igen Wachstum zwischen 1998 und 2002 liegt dieser Bereich um 4\% über dem Beschäftigungswachstum der Gesamtwirtschaft.

In Bezug auf die Musik gehört zu den wichtigsten Ergebnissen die Tatsache, dass die Wiener Musikwirtschaft in ein Zwei-Sektoren-System zerfällt. Im traditionellen klassischen und durch die öffentliche Mittel finanzierten Musikbereich verfügt Wien über eine gut ausgebaute Wertschöpfungskette, entsprechende Verwertungsbedingungen und professionelle Managementstrukturen. In dem weitgehend privatwirtschaftlich und kleinteilig organisierten U-Musik-Bereich hingegen existieren zwar maßgebliche Potentiale, die jedoch bis dato - auch aufgrund der Defizite im Bereich der Kapitalbildungsmöglichkeiten sowie der mangelnden branchenspezifischen Managementkapazitäten - kaum ökonomisch relevant umgesetzt werden. Für die Zukunft wird allerdings in diesem Bereich eine positive Entwicklung erwartet, weil

- die Beschäftigten hoch qualifiziert sind,

- die Cooperations- und Clusterstrukturen stark ausgebaut sind,

- die Hälfte der Unternehmen erst in den letzten zehn Jahren gegründet worden ist,

- die Unternehmen eine hohe Innovationsneigung aufweisen und

- die Nachfrageentwicklung positiv eingeschätzt wird (vgl. Ratzeböck et al. 2004: 12).

\section{Beispiele politischer Förderstrategien}

\section{Imagine a Toronto}

Die erwähnten sowie weitere Studien (vgl. z.B. Cliché et al. 2002) reflektierten nicht nur kritisch die Anfang der 1990er Jahre zunächst in Großbritannien einsetzende »Floridisierung « der Städte, d.h. die zunehmende Fokussierung der Politik auf kulturelle Sachverhalte, sondern sie beeinflussten auch die in der Zwischenzeit bereits weltweit angewandten politischen Förderstrategien. Im kanadischen Toronto stellt beispielsweise ein Expertenbericht mit dem Titel Imagine a Toronto - Strategies for a Creative City 
ein Schlüsseldokument der Urbanitätsentwicklung dar. ${ }^{6}$ Die Expertise, explizit auf Florida zurückgreifend, basiert auf dem Grundsatz »creativity means business « und teilt Floridas Überzeugung, es bestehe »a direct link between a flourishing city and the vitality of its creative sector «. Aus diesem Grund müsse Creativity als »the ultimative economic resource « betrachtet werden, sadding a new dimension to the competitive potential of cities around the world «.

Angelehnt an erfolgreiche Vorbilder - Imagine a Toronto ist ein Kooperationsprojekt mit der im Jahre 2004 vom Londoner Bürgermeister initiierten Strategic Agency for London's Creative Industries namens Creative London $^{7}$ - empfahlen Experten Investitionen in die ästhetische Bildung, die finanzielle Unterstützung von Creative Industries bzw. den erleichterten Zugang zum Creative Risk Capital in Form von günstigen Darlehen. Weitere Empfehlungen betrafen die Vernetzung bestehender Creative Clusters und den Ausbau von entsprechender Infrastruktur, insbesondere von den so genannten Creativity/Innovation Convergence Centres. Darunter wird die Ansiedlung von Kleinbetrieben aus unterschiedlichen ästhetischen Bereichen in unmittelbarer räumlicher Nähe verstanden, um die Zusammenarbeit zwischen den unterschiedlichen Sektoren zu inspirieren, neue Ideen zu generieren und diese zu vermarktbaren Produkten zu entwickeln. Unterstützt werden diese Bemühungen durch eine groß angelegte Kampagne unter dem Motto ${\text { Live with Culture }{ } .8}^{8}$

\section{Das Mannheimer Modell}

Wie das behandelte kulturpolitische Paradigma in der Bundesrepublik in die Praxis umgesetzt wurde, soll an den Beispielen Mannheim und Hamburg verdeutlicht werden. Das Mannheimer Modell basiert auf den vom badenwürttembergischen Staatministerium für Wirtschaft herausgegeben Empfehlungen zur Förderung der Popular- und Jugendmusik in Baden-Württemberg und geht von den folgenden Voraussetzungen aus:

»Kreativität ist ein entscheidender Rohstoff hochentwickelter Gesellschaften im 21. Jahrhundert. In den vielfältigsten Ausdrucksformen von Popmusik und Popkultur kommt dieses hohe Kreativpotential in beeindruckender Weise zum Ausdruck. Popmusik und Jugendkultur spielen jedoch auch eine wichtige Rolle für Gesellschaft, Kultur und Wirtschaft unseres Landes. Obwohl Popmusik seit über 50 Jahren viele unserer Lebensbereiche prägt und

6 Vgl. http://www.imagineatoronto.ca (Zugriff am 7.6.2007).

7 Vgl. http://www.creativelondon.org.uk (Zugriff am 7.6.2007).

8 Vgl. http://www.imagineatoronto.ca (Zugriff am 7.6.2007). 
beeinflusst, wird ihre Bedeutung vielfach auch heute noch unterschätzt. Von einer starken, lebendigen Kreativszene profitieren nicht nur die berührten Wirtschaftsbereiche. Sie kann auch ein Motor und Imageträger für das ganze Land sein. Es ist unser Ziel, Baden-Württemberg zu einem modernen, zukunftsorientierten Kreativstandort auszubauen« (Palmer 2002: 7).

Auf der Basis erwähnter Überlegungen wurde ein Gesamtkonzept für die Popförderung in Mannheim erarbeitet. Die erste Säule dieses Konzeptes bildet die Popakademie Mannheim. Hier wird ein dreijähriges Studium zum Bachelor of Arts in den Fächern Popmusikdesign und Musikbusiness angeboten. Darüber hinaus führt die Akademie die Fort- und Weiterbildung von Musiklehrern durch, unterhält einen Bandpool, der junge Musiker unterstützt und ihnen den Sprung ins Profi-Lager erleichtert, organisiert jährliche Branchenmeetings und kooperiert mit ähnlichen Institutionen im In- und Ausland mit der Zielsetzung, ein europaweites Popdesign-Curriculum zu entwickeln (vgl. Popakademie Mannheim 2004).

Die zweite Säule stellt der Musikpark dar, ein neu errichtetes Creativity/Innovation Convergence Centre, in dem diverse Studios, Labels und Promotion-Agenturen untergebracht sind, also Kleinunternehmen aus dem Bereich der Creative Industries (vgl. Stadtmarketing Mannheim 2004).

\section{Das Hamburger Musikhaus Karostar}

In Hamburg machte sich für die Creative Industries die Stadterneuerungsund Stadtentwicklungsgesellschaft Hamburg mbH stark, die Anfang 2006 das neu errichtete Musikhaus Karostar ihrem Zweck übergab. Dieses Zentrum für junge Unternehmen und Gründer aus der Musikbranche wurde nach englischen Vorbildern konzipiert und soll den Wandel der Hamburger Musikwirtschaft hin zu kleineren, flexibleren Unternehmen unterstützen. Das 5,4 Millionen Euro teuere Projekt wurde von der Europäischen Union und von der Hamburgischen Behörde für Wirtschaft und Arbeit finanziert.

Karostar bietet Gründern aus dem gesamten Musikbereich günstige Startkonditionen an, wie etwa niedrige Mieten und die Imagewirkung einer gemeinsamen Adresse. Sein Mieter-Mix liest sich wie ein Who is Who der jungen Hamburger Musikszene und deckt alle Sparten der Branche ab: von Plattenlabeln über den Vertrieb, Studios und Promotion-Agenturen bis hin zu TV- und DVD-Produktionsfirmen. Ferner zog ins Karostar die Firma »Hanseplatte - Musik von hier «, Hamburgs erstes lokales Musikgeschäft, das ausschließlich Tonträger, Printmedien, Filme und Merchandise-Produkte von Hamburger Musikschaffenden anbietet (vgl. Reuther 2006; STEG Hamburg $\mathrm{mbH} 2006)$. 
»Der Karostar trägt zur Aufbruchstimmung in der kulturellen Szene bei und verleiht der Hamburger Musikwirtschaft wichtige Impulse «, lobte die Kultursenatorin Karin von Welck das Projekt in ihrer Eröffnungsrede (zit. n. STEG Hamburg mbH 2006). Die zuvor nicht beachtete Creative Class in der Creative und Competitive City Hamburg ist zu Ehren gekommen und erfreut sich neben der politischen Unterstützung ${ }^{9}$ auch Einladungen zu Gesprächen mit Vertretern der wirtschaftlichen Prominenz, wie dies beispielsweise bei einer im Jahre 2005 vom Verleger Hubert Burda einberufenen Kreativrunde zum Thema »Ist Deutschlands kreative Klasse konkurrenzfähig?« der Fall gewesen ist. ${ }^{10}$ Auch die »Patriotische Gesellschaft von 1765 « hob in ihrer mit Hamburg - Wachsende Stadt betitelten Denkschrift unter dem Stichwort Wirtschaft die Musikindustrie als den einzigen Wirtschaftzweig hervor und betonte, die Hamburger Politik müsse sich

»insgesamt stärker bewusst werden, dass es sich bei der Musikindustrie um eine der wenigen Kultursparten handelt, die sich weitgehend ohne Subventionen selbst finanziert. Von daher hat die Populärmusik nicht nur eine große Bedeutung für die kulturelle Strahlkraft und damit die Attraktivität einer Stadt insbesondere für junge Leute, sondern sie hat auch als Wirtschaftszweig eine originäre ökonomische Relevanz. In beiderlei Hinsicht hat die Musikindustrie also eine erhebliche Bedeutung für das Leitbild der Wachsenden Stadt « (Patriotische Gesellschaft 2005: 35).

9 Insbesondere die Hamburger Grünen (GAL) sind in diesem Zusammenhang aktiv. Sie berufen sich in ihrem aktuellen Programm auf Richard Florida und verfolgen das Leitbild einer Kreativen Stadt: »In vielen Metropolen weltweit findet derzeit eine fundamentale Neuorientierung statt. Statt nur an Altbewährtem festzuhalten, setzen sie auf Innovationsvorsprung im globalen Wettbewerb der Regionen. Sie finden neue Wege für wirtschaftliche, soziale und ökologische Herausforderungen. Sie heben die ungenutzten Potenziale ihrer Stadt und vor allem ihrer Menschen. Sie sind zur >kreativen Stadt - geworden« (vgl. http:// www.hamburg-kreativestadt.de; Zugriff am 20.6.2007). Im Kulturbereich setzen die Grünen in erster Linie auf Creative Industries. Sie beriefen ein Hearing mit den Hauptprotagonisten der Hamburger Musikszene ein und erarbeiteten ein Gesamtkonzept zur Förderung der Hamburger Kreativwirtschaft (vgl. »Hafen für Ideen. Kreativwirtschaft stärken. Handlungsvorschläge der GAL«; per E-Mail versandtes Paper).

10 Vgl. http://www.hubert-burda.de/archives/2005/10/erfolgreicher_s.html, eingestellt am 25.10.2005 (Zugriff am 14.6.2007). 


\section{The Brave New Creative Era?}

Abschließend sollen noch einige Anmerkungen gemacht werden, wie die in groben Zügen skizzierte Entwicklung einzuschätzen ist.

1. Floridas Feststellung, das ökonomische Wachstum hänge von der Creative Class ab (vgl. Florida 2004b und 2004a), ist lobenswert, weil er als Wirtschaftswissenschaftler ins Zentrum seiner Ausführungen nicht Rohstoffe, Firmen und Finanzen, sondern die Menschen stellt. Er betrachtet sie als Individuen, die sich durch die Eigenschaften »individuality, selfexpression und openess to diversity« (Florida 2004b: 9) auszeichnen, sich aber ihrer Klassenzugehörigkeit noch nicht bewusst sind. Sein Aufruf gilt deshalb der Bildung einer Klasse kreativer Individuen im Sinne einer Vor-

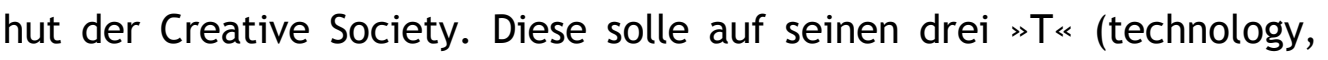
talent, tolerance) basieren und trotz ihrer festen Verwurzelung im Ökonomischen einen »experimential lifestyle« (ebd.: 13) fördern.

2. Viele Ideen, die mit Florida assoziiert werden, sind keineswegs neu, denn Überlegungen über die Bohème, die Rolle der Kreativität und die Bedeutung der Kreativen für die künftige Entwicklung der Wirtschaft einer Stadt, einer Region oder eines Staates haben sich bereits zahlreiche Forscher vor ihm gemacht. Auch die Inszenierung der Urbanität durch die Kultur bzw. die so genannte Kulturalisierung der Städte hat bereits längst vor Florida begonnen (vgl. Häußermann/Siebel 1993; Bittner 2001). Mit Floridas Bestseller The Rise of the Creative Class fanden diese Gedanken allerdings eine breite Leserschaft auch außerhalb der Fachkreise und bilden zurzeit das dominierende Paradigma, auf dem eine Neubewertung des Kulturbereiches durch die Politik basiert. Nicht zuletzt auch deshalb, weil Florida - ähnlich wie Charles Landry in seinem Buch The Creative City. A Toolkit for Urban Innovators (2000) konkrete Vorschläge für die Umsetzung theoretischer Überlegungen in die Praxis liefert. ${ }^{11}$

3. Florida selbst legt zwar großen Wert auf das Bestehen einer lebendigen Musik- und Kunstszene, betrachtet sie aber weniger als eine ökonomische Variable sui generis, sondern vor allem als einen wichtigen Stand-

11 Ebenso wie Richard Florida gilt auch der Brite Charles Landry als international anerkannte Autorität für Stadterneuerung und Kreativität. Gleichfalls unterhält er eine Beratungsfirma, die zahlreiche Projekte in In- und Ausland durchführte, darunter in Albanien, Australien, in der Schweiz und in Japan (vgl. http:// www.charleslandry.com/index.php?l=proj_proj; Zugriff am 20.6.2007). 
ortfaktor, der andere Kreative, insbesondere aus dem Hi-Tech-Bereich, an eine Stadt zu binden imstande sei (vgl. Florida 2004a: 128). Oder besser gesagt: seiner Ansicht nach gibt es zwischen der künstlerischen und der wissenschaftlichen Kreativität keine nennenswerten Unterschiede, da sich diese beiden Formen der kreativen Arbeit gegenseitig bedingen (Florida 2004b: 55). Trotzdem fielen seine Gedanken insbesondere im Bereich der Popmusik, einem der wichtigsten Content-Lieferanten der Creative Industries, auf einen besonders fruchtbaren Boden. Sie werden einerseits im Zusammenhang mit Sachverhalten wie »Musik als Wirtschaftsfaktor « und »Kultur als Zukunftsbranche«, d.h. in einem ökonomischen Kontext, andererseits in Hinblick auf die Rolle der populären Musik für das Image-Design einer Stadt bzw. für die strategische Bedeutung lokaler Soundscapes im Wettbewerb zwischen den Creative Cities diskutiert.

Soziale Aspekte, wie etwa die Bedeutung der Musik für die Konstruktion unterschiedlicher Lifestyles und die Bildung divergenter Identitätsentwürfe, also Sachverhalte, die von Florida wiederholt unter dem Aspekt der Toleranz hervorgehoben werden, spielen bei den erwähnten Überlegungen eine sekundäre Rolle. Es ist aber zu einem veränderten Verhältnis zu dem zuvor entweder nicht beachteten oder an den gesellschaftlichen Rand abgeschobenen musikalischen Underground gekommen, der nun als ein wichtiger Content Lieferant betrachtet wird.

4. Die Förderung der Creative Industries steht zurzeit auf der politischen Agenda, sowohl in den kulturellen Richtlinien der UNESCO ${ }^{12}$ und der Europäischen Union (vgl. Cliché et al. 2002) als auch in vergleichbaren Dokumenten auf regionaler Ebene. Offen bleibt jedoch die Frage, ob sich internationale Politikkonzepte ohne weiteres auf konkrete Bedingungen übertragen lassen bzw. ob die Anwendung gleichartiger Förderstrategien auf globaler Ebene dem Erhalt der kulturellen Vielfalt und der unverwechselbaren Eigenidentität lokaler Kulturen dienlich ist.

12 Mit der Übernahme der Thesen über die Kreativität als Rohmaterial und den Beitrag der Creative Industries zu Wohlstand und Beschäftigung treibt die UNESCO die Kommerzialisierung der Kultur erbarmungslos weiter und beeinflusst weltweit die angewandten politischen Förderstrategien. Ferner rief die UNESCO ein Creative Cities Network ins Leben mit der Zielsetzung, die Rolle der einzelnen Städte als »centres of excellence« zu bestätigen sowie anderen Städten, insbesondere in den Entwicklungsländern, bei der Förderung der jeweiligen Creative Economy zu helfen (vgl. http://portal.unesco.org/culture/ en/ev.php-URL_ID=2461\&URL_DO=DO_TOPIC\&URL_SECTION=201.html; Zugriff am 19.5.2007). 
5. Nicht alle bislang angewandten Fördermaßnahmen erfüllen die hoch gestochenen Erwartungen. Barcelona verbuchte mit seiner konsequenten Creative Policy zwar signifikante Erfolge. ${ }^{13}$ In Großbritannien hingegen, wo Tony Blair im Rahmen seiner Cool Britannia-Kampagne eine Creative Industries Task Force ins Leben rief und wo die Förderung der Creative Industries zum Zweck der urbanen Regeneration am konsequentesten betrieben wird, fielen Ergebnisse der gezielten Musikförderung unterschiedlich aus. Die Stadt Liverpool, die ihren Flughafen in »John Lennon Airport « umtaufte, profitierte von ihrem Image als Beatles City, und das Cavern Quarter, das sich als Künstlerviertel zu inszenieren versucht, lockt massenhaft Touristen an. Das Geschäft mit der musikalischen Vergangenheit blüht, wogegen sich andere Musiksegmente weder der entsprechenden Aufmerksamkeit noch des ökonomischen Aufschwungs erfreuen können. Auch das von Paul McCartney initiierte Liverpool Institute for Performing Arts trug nicht im erhofften Maße zur Gestaltung der Liverpooler Szene bei, da es in erster Linie eine internationale studentische Klientel anlockt, die nach dem Studium die Stadt wieder verlässt (vgl. Cohen 2007: 130ff.).

Pläne für die Anerkennung von Sheffield als kulturelles Zentrum scheiterten vollkommen. Dort wurde ein Cultural Industries Quarter errichtet, dem unter anderen das 1999 eröffnete National Centre for Popular Music, ein Popmusik-Museum, angehörte: Die Kosten in Höhe von 15 Millionen Pfund wurden von der Staatslotterie und dem Entwicklungsfond der Europäischen Union getragen. Der erwartete Besucherstrom blieb allerdings aus, das Museum wurde geschlossen und das Gebäude für andere Zwecke freigegeben (vgl. Anon. 1999). Gründe dafür dürften neben der mangelnden Zusammenarbeit mit den lokalen Akteuren auch darin gelegen haben, dass sich die Szene von Sheffield, bekannt vor allem durch Human League, nicht ähnlich vermarkten ließ wie Liverpool mit seinen mehrheitsfähigen Beatles.

6. Eine weitere Unsicherheit betrifft die Frage, ob eine Musikszene überhaupt einer proaktiven bzw. interventionistischen Politik seitens der Stadt bedarf. Eine Untersuchung von Geoff Stahl über Montreal (vgl. den Beitrag in diesem Band) hat nämlich gezeigt, dass die lebendige Musikszene dieser Stadt ihre Impulse weder aus der städtischen Förderung noch aus ihrer wirtschaftlichen Stärke generiert. Ganz im Gegenteil: Die ökonomische Lage von Montreal ist schlecht, die Armut groß. Ein zusätz-

13 http://www.kulturmanagement.net/downloads/barcelona-mascarell.pdf (Zugriff am 10.6.2007). 
liches Problem bilden die Spannungen zwischen der frankophonen Mehrheit und der anglophonen Minderheit. Trotzdem zieht die Stadt vor allem junge kreative Individuen an und hat ein ähnlich positives Image einer Kunst- und Kulturstadt wie Paris oder Berlin.

Ausschlaggebend dafür sind laut Stahl die folgenden Faktoren: Wegen der schlechten Wirtschaftslage und der daraus resultierenden Abwanderung gibt es viel billigen Wohnraum, insbesondere in dem als Plateau bekannten Künstlerviertel. Ferner verlor das ehemalige Industriegebiet Old Port mit der zunehmenden De-Industrialisierung seine wirtschaftliche Basis. Was geblieben ist, sind alte Warenhäuser und andere Gebäudeflächen, die von Künstlern als Arbeitsplätze genutzt werden können.

Einen wichtigen Aspekt bildet ferner die Tatsache, dass die vier in Montreal ansässigen Universitäten die niedrigsten Studiengebühren in ganz Nordamerika erheben, was zahlreiche Studenten anlockt. Viele von ihnen bleiben und bilden eine neue soziale Schicht, die Geoff Stahl als »overeducated« und »underemployed« umschreibt. Und last but not least spielt auch die »cultural mythology« dieser Stadt eine wichtige Rolle. Montreal ist als Kanadas Sündenstadt bekannt, und sie zieht bereits seit Anfang des 20. Jahrhunderts Jazzmusiker, Schriftsteller, Maler und Filmemacher an, die wiederum - ähnlich wie die Pariser Bohème - in ihren Werken der Stadt ihre Aura verleihen.

Trotz der ungünstigen wirtschaftlichen Verhältnisse herrscht in Montreal ein attraktives soziales Klima, sodass für Musiker und andere Künstler das Plateau auch keinen sozialen Brennpunkt, sondern eine Enklave der Bohème mit romantisch-utopischen Sinnzuweisungen darstellt. Was im euphorisch geführten politischen Diskurs über Creative Class, Creative Industries und Creative City viel zu kurz kommt, ist deshalb das Eingehen auf Bedürfnisse, Zielsetzungen und Erwartungshaltungen der unmittelbar Betroffenen, denn die meisten unter ihnen verstehen die musikalische Tätigkeit nicht vorrangig als einen gewinnbringenden Wirtschaftsbereich, sondern als einen erstrebenswerten »way of life« (vgl. Cohen 2007: 109). 


\section{Literatur}

Anon. (1999). »Debts Rock Pop Museum.«In: BBC News, 18. Oktober, http://news. bbc.co.uk/2/hi/entertainment/478616.stm (Zugriff am 7.7.2007).

Bittner, Regina (2001). Die Stadt als Event. Zur Konstruktion urbaner Erlebnisräume. Frankfurt/M.: Campus-Verlag.

Cliché, Danielle / Mitchell, Ritva / Wiesand, Andreas (2002). Creative Europe: On Governance and Management of Artistic Creativity in Europe. Bonn: ARCult Media.

Cohen, Sara (1991). »Popular Music and Urban Regeneration: The Music Industries on Merseyside«. In: Cultural Studies 5, Nr. 3, S. 332-346.

Cohen, Sara (2007). Decline, Renewal and the City in Popular Music Culture: Beyond the Beatles. Aldershot: Ashgate.

Florida, Richard (2004a). Cities and the Creative Class. London: Routledge.

Florida, Richard (2004b). The Rise of the Creative Class: And how it's Transforming Work, Leisure, Community and Everyday Life. Cambridge, MA: Basic Books.

Häußermann, Hartmut / Siebel, Walter (Hg.) (1993). Festivalisierung der Stadtpolitik. Stadtentwicklung durch große Projekte (= Leviathan Sonderheft 13). Opladen: Westdeutscher Verlag.

Hartley, John (Hg.) (2005). Creative Industries. Singapore: Blackwell.

Horkheimer, Max / Adorno, Theodor W. (1971). Dialektik der Aufklärung. Philosophische Fragmente. Frankfurt/M.: Fischer.

Howkins, John (2002). The Creative Economy: How People Make Money from Ideas. London: Penguin.

Landry, Charles (2000). The Creative City: A Toolkit for Urban Innovators. London: Earthscan Publications.

Lloyd, Richard (2006). Neo-Bohemia. Art and Commerce in the Postindustrial City. New York, Oxon: Routledge.

O'Connor, Justin / Banks, Mark (2000). Cultural Industries and the City: Innovation, Creativity and Competitiveness. Provisional Report for the Economic and Social Research Council, British Cities Programme. Unveröffentlichtes Manuskript.

Palmer, Christoph E. (Hg.) (2002). Empfehlungen der Arbeitsgruppe: Förderung der Popular- und Jugendmusik in Baden-Württemberg. Stuttgart: Staatsministerium Baden-Württemberg.

Patriotische Gesellschaft (Hg.) (2005). Hamburg - Wachsende Stadt. Eine Denkschrift der Patriotischen Gesellschaft von 1765. Hamburg: Patriotische Gesellschaft.

Popakademie Mannheim (2004). Promotionmappe zum POP.FORUM: branchen. meeting. Mannheim: Popakademie Mannheim.

Raunig, Gerald (2007). »Kreativindustrie als Massenbetrug. «In: Kritik der Kreativität. Hg. v. Gerald Raunig und Ulf Wuggenig. Wien: Turia + Kant, S. 67-78.

Ratzenböck, Veronika et al. (2004). Untersuchung des ökonomischen Potentials der "Creative Industries « in Wien. Endbericht, erstellt von Kulturdokumentation, Mediacult und Österreichischem Institut für Wirtschaftsforschung im Auftrag des Magistrats der Stadt Wien, MA 27, der Wirtschaftskammer Wien und des Filmfonds Wien. Wien: WIFO, http://www.creativeindustries.at/pdf/Endbericht.pdf (Zugriff am 24.6.2007). 
Reuther, Birgit (2006). »Die Branche zieht ein - mit Platten und Trompeten.« In: Hamburger Abendblatt, 27. Januar, http://www.abendblatt.de/daten/2006/ 01/27/527431.html (Zugriff am 10.6.2007).

Schüle, Klaus (2003). Paris: die kulturelle Konstruktion der französischen Metropole: Alltag, mentaler Raum und sozialkulturelles Feld in der Stadt und in der Vorstadt. Opladen: Leske + Budrich.

Stadtmarketing Mannheim (2004). Mit dem >Mannheimer Modelk in die Zukunft des Pop. Presseinformation vom 1 . Juni.

STEG Hamburg mbH (2006). Die Hamburger Musikbranche feiert den Start des Karostar Musikhauses St. Pauli. Pressemitteilung der Stadterneuerungs- und Stadtentwicklungsgesellschaft Hamburg mbH vom 20. Februar, per E-Mail versandtes Paper.

\begin{abstract}
Currently, the political discourse on the sound and the city is oscillating around the catchwords Creative Class, Creative Industries and Creative City. Under neo-liberal political conditions creativity is understood as an economic resource with an almost unlimited growth potential. Consequently, it is a vital interest of a city to foster its creative capital in order to generate wealth and employment on the economic level as well as to increase its symbolic value in the global competition of urban conglomerates. Applied to popular music, this paradigm encouraged the financial support of certain parts of the music infrastructure and the foundation of new socalled creativity/convergence centres. However, as empirical studies proved, the political strategies applied do not necessarily achieve anticipated outcomes, since the top-down approach as promoted especially by the UNESCO and the European Union, does not take into account the regional characteristics of actual musical scenes and the intentions, needs and expectations of the individuals and institutions involved.
\end{abstract}

